\title{
Fluorescent Detection and Isolation of DNA Variants Using Stabilized RecA-Coated Oligonucleotides
}

\author{
Michael C. Rice, ${ }^{1}$ Brandy M. Heckman, ${ }^{1}$ Yi Liu, $^{2}$ and Eric B. Kmiec ${ }^{1,3}$ \\ ${ }^{1}$ Department of Biology, Delaware Biotechnology Institute, University of Delaware, Newark, Delaware 19711, USA; ${ }^{2}$ NaPro \\ Genomics, Newark, Delaware 19711, USA
}

\begin{abstract}
Several genome resequencing strategies have been developed to detect genetic variation in populations and correlate diversity with phenotypic consequences. Commonly used methods of detecting single nucleotide polymorphisms (SNPs) use PCR amplification and indirect analysis, which can create template biases and enable user contamination. Here we present a novel assay to detect and isolate DNA variants using stabile nanostructures formed directly on duplex DNA. The assay incorporates the well-established RecA-catalyzed strand invasion process with a novel stabilizing hybridization step. First, short RecA-coated oligonucleotide filaments invade duplex DNA to form a synaptic intermediate or "D-loop." Sequentially, chemically modified oligonucleotide probes anneal to the displaced DNA strand of the complex to form a stable "double D-loop." These joint molecules resist dissociation when both oligonucleotides are completely complementary to the target duplex; however, if the probes are mismatched, the complex is inherently instable and rapidly dissociates. SNPs are identified by detecting the fluorophore assimilated into stable complexes produced by homologous probes compared to unstable differentially labeled mismatched probes. Furthermore, this strategy can be used to isolate specific allelic variants by affinity purification from complex populations. Stabilized double D-Loop intermediates accordingly offer the promise of haplotyping and pharmacogenomic analysis directly in double-stranded DNA samples.
\end{abstract}

Information gained from genome sequencing initiatives has been organized into interactive databases, which can be used to profile individual DNA samples and detect variations within and among populations (Smigielski et al. 2000; Daly et al. 2001; Gabriel et al. 2002). In their simplest form, gene variations known as single nucleotide polymorphisms (SNPs) are used as markers to create a genetic linkage map. The molecular etiology of many human diseases is often associated with specific genomic landmarks connecting phenotype with a definable location on a chromosome (Riley et al. 2000). At the level of the genotypes, SNP-maps can facilitate the establishment of haplotypes, which enable a statistical measurement of allelic variation within complex traits.

Standard methods of genotyping use PCR amplification of template DNA to initialize the analysis. PCR requires little starting material so that many markers can be multiplexed using small amounts of template. The genotype is then compiled by RFLP, ASPCR, direct sequencing, ligation, differences in microsatellite size, TaqMan molecular beacons, or dideoxy terminator primer extension (for reviews, see Kirk et al. 2002; Shi 2002). Thus, these genotyping techniques, albeit indirect, can give accurate results from an amplified DNA target template. Exponential amplification, however, requires additional steps and specimen handling, which can increase the potential for user contamination (Burkardt 2000; Millar et al. 2002). The potential for these errors has prompted investigators to explore alternative methodologies that query the sample more directly. To fill this void, technologies that can detect SNPs directly from genomic DNA, such as the Invader Assay (Lyamichev et al. 1999; Ryan et al. 1999) and Rolling Circle Amplification (RCA; Lizardi et al. 1998; Zhong et al. 2001), have been developed. These assays use an isothermal linear amplification method to detect sequence variations. The Invader assay uses mismatch-specific DNA cleav-

\section{${ }^{3}$ Corresponding author.}

E-MAIL ekmiec@udel.edu; FAX (302) 831-3427.

Article and publication are at http://www.genome.org/cgi/doi/10.1101/ gr.1386204. Article published online before print in December 2003. age products, which release fluorophore quenching to emit a fluorescent signal, whereas RCA uses mismatch-dependent ligation to facilitate polymerization and branching of fluorescent oligonucleotides.

In a different approach, novel assay systems engaging recombinase-coated probes have been used to select specific duplex DNA substrates from a heterogeneous population (Sena and Zarling 1993). The reaction takes advantage of the activity of the RecA protein, which polymerizes on single-stranded DNA to form a right-handed helical nucleoprotein filament capable of invading duplex DNA in a sequence-independent fashion. The RecA-coated nucleoprotein filament scans the duplex for homologous sequence and, once located, mediates strand exchange and recombination between the incoming DNA strand and the duplex (Cunningham et al. 1979). In the presence of the slowly hydrolyzable ATP analog, ATP- $\gamma \mathrm{S}$, the search stops when regions of homology fall into alignment, establishing a complex with the nucleoprotein filament bound to the duplex with the concurrent displacement of the homolog strand; this joint structure is commonly referred to as a "displacement loop" (D-loop; Shibata et al. 1979). As a result, the displaced strand becomes available and is capable of hybridizing a second single-stranded nucleic acid molecule to create a four-stranded joint molecule known as a "double D-loop" (dD-loop; Jayasena and Johnston 1993). RecA-mediated homology searches are permissive to mismatches, tolerating up to $30 \%$ heterology, with probes having lengths of $>200 \mathrm{bp}$ (Morel et al. 1994). But, when the probe size is substantially decreased, tolerance of heterology is correspondingly, and significantly, eliminated.

We have taken advantage of this heterology intolerance to develop a novel assay for detecting SNPs directly in mixed samples of duplex DNA, as a potentially useful initial step in a genotyping regime. Here, we introduce an in vitro diagnostic method that relies on short oligonucleotide probes that form synaptic intermediates of the recombination process in a homologous target. DNA variants are fluorescently detected precisely and isolated from a library of gene fragments. With this 
method, it is possible to separate allelic sequences and assay for haplotypes directly.

\section{RESULTS}

\section{Fluorescent Detection of Specific DNA Sequences Using Double D-Loops; Synaptic Intermediates}

The assay system presented here was originally devised and used as an in vitro system to model intermediates in gene repair reactions (Liu et al. 2001, 2002; Parekh-Olmedo et al. 2002). In vivo, single-stranded oligonucleotides pair with a homologous target and form three-stranded intermediates that contain a central, single base heterology (for review, see Brachman and Kmiec 2002). This central mismatch purportedly activates endogenous repair pathways and the intermediate structure is resolved. In vitro, the intermediate, however, can be stabilized by a fourth strand comprised of a modified annealing oligonucleotide that hybridizes to the outgoing strand of the target duplex DNA. Detection of such repair intermediates was first accomplished with ${ }^{32}$ P-labeled oligonucleotides and short, duplexed oligonucleotide substrates (Gamper et al. 2003). A schematic of the assay developed to detect specific DNA sequences and altered sequences is presented in Figure 1. First, a DNA "incoming" oligonucleotide is coated with RecA in the presence of a slowly hydrolyzable ATP analog, ATP $\gamma \mathrm{S}$, and is then added to a duplex target DNA. The single D-loop intermediate is unstable in forms of DNA lacking a substantial number of supercoils; however, the addition of a second modified "annealing" oligonucleotide, complementary to the displaced strand, creates a complement-stabilized double Dloop (dD-loop), or synaptic complex. These structures, formed by oligonucleotides of appropriate size and modifications, significantly increase the stability of the intermediate.

This method is capable of forming a stable dD-loop complex with various DNA topologies. We used our standard assay to test plasmid DNA substrates that were superhelical (sc), topoisomerase I relaxed covalently closed circular (ccc), and restriction endonuclease linearized (lin) for the ability to form stabilized double D-loops. Cyanine dyes, which can be detected at different excitation and emission wavelengths so that each fluorophore can be independently visualized, were used to monitor single and double D-loop formations. Cy5 intensities are detected at $670 \mathrm{~nm}$ and are designated red, whereas $\mathrm{Cy} 3$ intensities are detected at $555 \mathrm{~nm}$ and are designated green. In cccDNA and linDNA, the complementary annealing oligonucleotide is vital for the detection of a fluorescent $\mathrm{dD}$-loop complex as single D-loops are unstable, if formed at all, under these conditions. However, in supercoiled plasmid DNA, we observe an enhanced level of stability of fluorescent single D-loops without the need for stabilization by the annealing oligonucleotide. This is consistent with previous reports that the torsional strain of negative supercoils enables single D-loop formation (Wiegand et al. 1977). D-loop or dDloop complexes were examined by using either single oligonucleotides or sets of oligonucleotides with a Cy5-labeled incoming or annealing oligonucleotide. By measuring the relative intensities of the Cy5 fluorescence, we found that double D-loops are efficiently formed in any duplex DNA topology; however, in supercoiled plasmid DNA, single D-loop complexes are somewhat stable and $\mathrm{dD}$-loop complexes may be differentiated if the label is on the annealing rather than the incoming oligonucleotide.

Gamper et al. (2003) showed that modified annealing oligonucleotides with more RNA-like backbones such as LNA and 2 '-O-MeRNA are also effective in stabilizing a double D-loop joint in " $\mathrm{Y}$ " arms at duplex ends. We extended these observations in our assay system using longer, more complex duplex targets. An-
Incoming Oligo

Add Recombinase / ATP $\gamma$ S

Recombinase Filament (5 min)

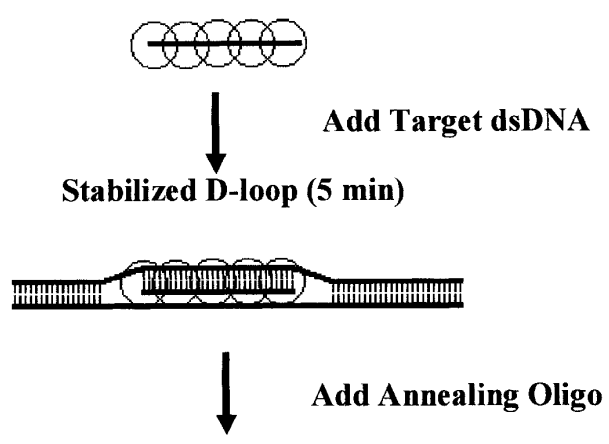

Stabilized double D-loop (1 min)

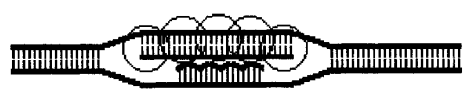

Protein denaturation

\section{Complement Stabilized D-loop (1 min)}

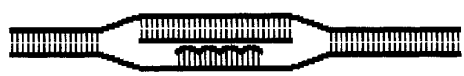

Figure 1 Schematic of standard double D-Loop formation assay. (1) A DNA "incoming" oligonucleotide is coated with RecA in the presence of a nonhydrolyzable ATP analog, ATP $\gamma$ S. (2) The preformed nucleofilament is added to the duplex target substrate, and RecA catalyzes the sequencedependent pairing and strand invasion of the duplex target, displacing one strand of the Watson-Crick base duplex to create a D-loop intermediate. (3) The D-loop intermediate is stabilized upon the addition of a second modified "annealing" oligonucleotide that is complementary to the displaced strand. (4) The resulting complement-stabilized double Dloop is analyzed after denaturing and dissociating RecA by adding SDS.

nealing oligonucleotides with different modified backbones were tested for their capacity to stabilize the joint molecule in linDNA. Modifications include DNA with phosphorothioate linkages (SDNA), "locked" nucleic acid residues (LNA), peptide nucleic acids (PNA), and 2'-O-methyl RNA (2'-O-MeRNA). A comparison of these modifications revealed a similar hierarchy of effectiveness as reported in Gamper et al. (2003): PNA $\geq 22^{\prime}-O$ $\mathrm{MeRNA} \geq \mathrm{LNA} \gg \mathrm{SDNA}>$ DNA (data not shown). In general, backbone modifications that are more DNA-like are less effective at stabilizing the complex. We have not determined, as yet, if this is caused by an enzymatic process of RecA or a decreased thermodynamic stability of DNA and phosphorothioate-linked DNA. This does, however, support the hypothesis that DNA backbone modifications block the reverse strand transfer reaction of RecA.

\section{Concurrent Detection}

The incoming and annealing oligonucleotides can both be labeled and tested in the dD-loop reaction. Figure 2 shows the formation of a double D-loop in a linear 8 -kb plasmid target with simultaneous detection of differentially labeled incoming and annealing oligonucleotides. No band in lane 1 displays the in- 


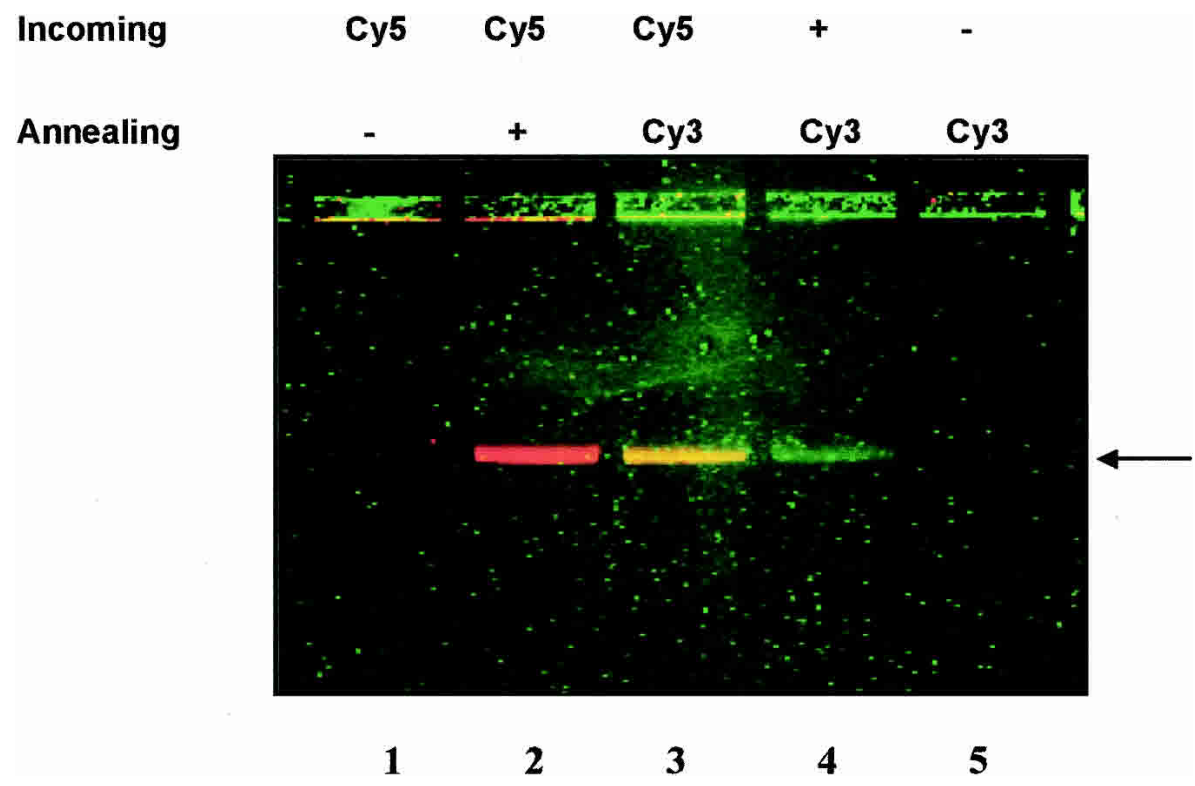

Figure 2 Concurrent detection of incoming and annealing oligonucleotides. The fluorescent gel picture shows the formation of a dD-loop in a linear 8-kb plasmid target with concurrent detection of differentially labeled incoming and annealing oligonucleotides. The incoming oligonucleotide is either unlabeled (no color) or Cy5-labeled (red). The incoming oligonucleotide is either unlabeled (no color) or Cy3-labeled (green) as indicated. (Lane 1) A Cy5-labeled incoming is unable to form a stable single D-Loop when no annealing oligonucleotide is added (no band). (Lane 2) Stabilization by adding a Cy5-labeled incoming and unlabeled annealing oligonucleotide in which the Cy5-labeled dD-loop is visualized as a red band. (Lane 3) Stabilization by adding a Cy5-labeled incoming and a Cy3-labeled annealing oligonucleotide in which both the incoming and annealing labels are colocalized and visualized as a yellow band combining red (Cy5) and green (Cy3) labels. The green band in lane 4 is created using an unlabeled incoming oligonucleotide and a stabilizing Cy3-labeled annealing oligonucleotide. (Lane 5) RecA is unable to form a stable single D-loop with a Cy3-labeled annealing oligonucleotide when no DNA incoming oligonucleotide is added (no band). The arrow indicates the position of the double D-loop as detected by fluorescence.

ability of a Cy5-labeled incoming to form a stable single D-loop when no annealing oligonucleotide is added. However, when an unlabeled annealing oligonucleotide is added, the Cy5-labeled double D-loop is visualized as a red band as seen in lane 2 . When a Cy3-labeled annealing is added, both the incoming and annealing labels are visualized as a yellow band combining red and green labels as in lane 3 . When an unlabeled incoming oligonucleotide is used in combination with a Cy3-labeled annealing oligonucleotide, a green band is visualized as in lane 4; and when no incoming oligonucleotide is used and only RecA and a Cy3labeled modified annealing oligonucleotide are added to the target, no band is seen in lane 5 , indicating no stable joint molecule is formed.

\section{Temperature Dependence}

Our model of double D-loop formation includes two distinct steps: an enzymatic strand invasion and a nonenzymatic DNA hybridization. The first step relies on the physiological properties of RecA to catalyze strand invasion, but the second step is driven by the thermodynamic properties of hybridization. To optimize these two phases concurrently, we compared the effect of increasing the reaction temperature of each step of double D-loop formation to test the yield of joint molecule formation (Fig. 3). Minimal formation of double D-loops (indicated by arrows) can be seen with incubations below $37^{\circ} \mathrm{C}$, with increasing formation from $37^{\circ} \mathrm{C}$ to $55^{\circ} \mathrm{C}$; and at $65^{\circ} \mathrm{C}$, formation is reduced. Thus, the optimal temperature for double D-loop formation is $50^{\circ}-55^{\circ} \mathrm{C}$ when a 31-mer incoming oligonucleotide and a 15-mer LNA- containing annealing oligonucleotide are used as this target. It was surprising that both steps of the reaction were optimized at higher temperature because RecA has a natural physiological temperature of $37^{\circ} \mathrm{C}$, whereas hybridization is increased at $55^{\circ} \mathrm{C}$. We observe higher yields of stable joint molecules, however, when both steps of the reaction are performed at $55^{\circ} \mathrm{C}$.

\section{Detection of Single Nucleotide} Polymorphisms With Fluorescently Labeled Incoming Oligonucleotides We observed that double D-loop complexes in which the labeled incoming oligonucleotide is mismatched to the template are significantly less stable after denaturing the RecA filament than complexes in which the oligonucleotide is perfectly complementary to the template. Fluorescent tags were placed on the incoming oligonucleotides using Cy5 (red) on the corresponding mutant and Cy3 (green) on the wild-type sequence. If both the oligonucleotides are mismatched, no detectable stable complex is detected. Accordingly, it is possible to add a mixture of differentially labeled fluorescent oligonucleotide probes that are homologous to different variants of a gene creating a multiplex detection reaction. In this competitive reaction, only the homologous $\mathrm{dD}$-loop complexes are stable and yield the corresponding fluorescent signal indicating polymorphic nucleotide. A genotype of a diploid organism could be determined by detecting the fluorescently labeled incoming oligonucleotides incorporated into the complex: Cy3 (green) for homozygous wild type, Cy5 (red) for homozygous mutant, or yellow for a heterozygote.

\section{Optimization of Labeled Incoming and Unlabeled Annealing Oligonucleotide Size Combinations}

An important consideration for effective SNP detection using this technique centers around the combination of optimized sizes of incoming oligonucleotide and annealing oligonucleotides. We examined size combinations that produce the most accurate scoring; the results are shown in Figure 4. The ability to discriminate mismatches is strongly dependent on the size of both the incoming and annealing oligonucleotides, providing a narrow range for this reaction. Under the conditions tested, the incoming oligonucleotide must be at least 23 nucleotides (nt) in length, ensuring that stable D-loop formation and sufficient displacement of the outgoing strand occur, which will increase the potential for hybridization of the annealing oligonucleotide. The upper size limit for the incoming oligonucleotide for precise genotyping is $35 \mathrm{nt}$; however, incoming oligonucleotides of 39 nt or larger give a mixed signal, losing mismatch fidelity. We submit that, under the conditions tested, the annealing oligonucleotide must be $>13 \mathrm{nt}$ in length to form stable double Dloops, and can be as large as $21 \mathrm{nt}$. A 31-mer incoming oligonucleotide and 17-mer annealing oligonucleotide are optimal; both for yield of double D-loops, as well as exhibiting a strong fidelity of mismatch discrimination.

\section{Genome Research} www.genome.org 


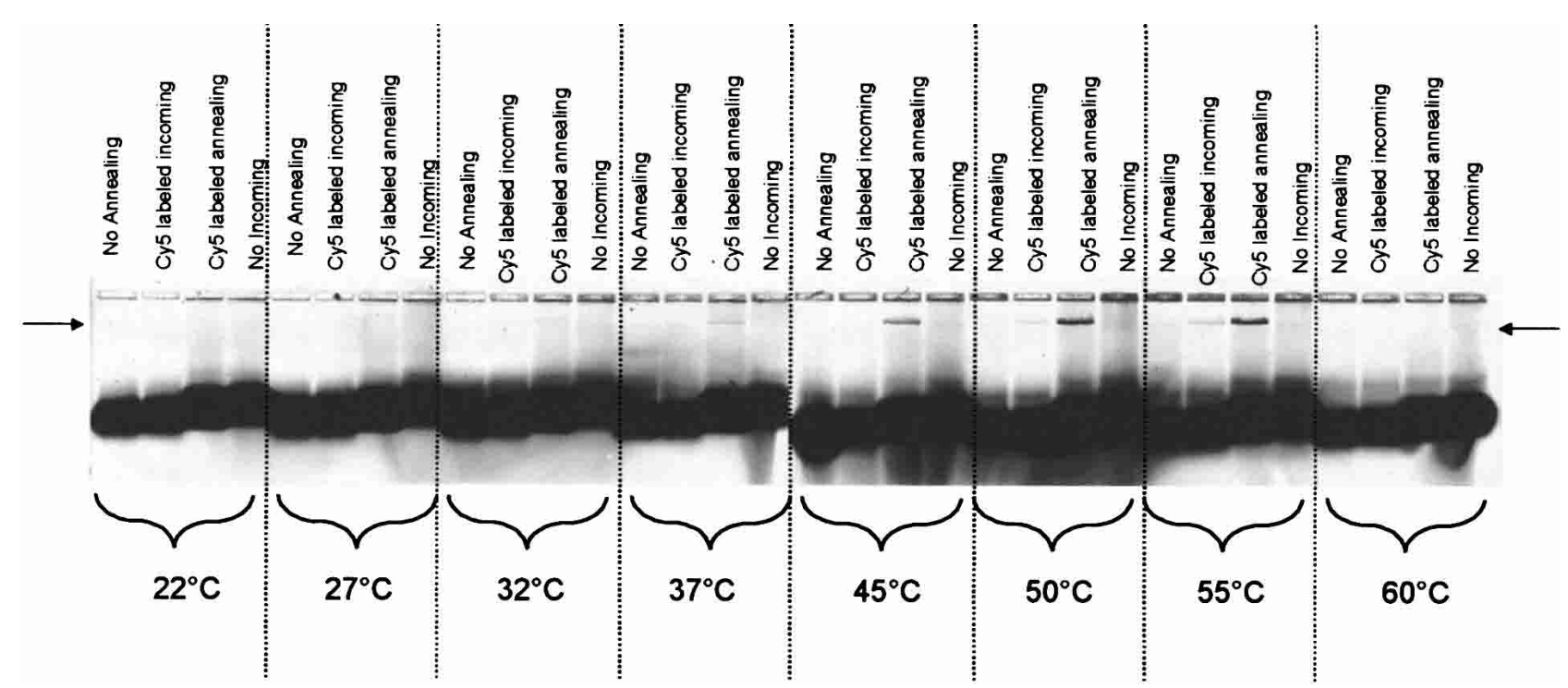

Figure 3 Thermostability and double D-loop yield. The gel compares the incubation temperature effects on the yield of joint molecule formation. Sets of four reactions were incubated at each of the temperatures indicated above. In the first reaction of each temperature set, Cy5-labeled incoming oligonucleotides are used alone to show the need for the annealing oligonucleotide. In the second reaction of each set, Cy5-labeled incoming is used with an unlabeled 15-mer LNA annealing oligonucleotide. In the third reaction of each set, unlabeled incoming is used with a Cy5-labeled LNA annealing oligonucleotide. And in the fourth reaction of each set, the Cy5-labeled annealing is used without an incoming oligonucleotide to show the need for the RecA filament. The arrow indicates the position of the double D-loop complex as detected by fluorescence.

\section{Optimization of Unlabeled Incoming and Labeled Annealing Oligonucleotides Based on Length}

We observe superior mismatch discrimination, however, when the fluorescent label is on the annealing oligonucleotide. In contrast to what we see with labeled incoming oligonucleotides, obvious mismatch discrimination is observed, regardless of the topology of the DNA target, with linear and supercoiled DNA targets showing precise mismatched discrimination (data not shown). Size is an important consideration when using labeled annealing oligonucleotides to accurately detect the correct genotypes. We examined size combinations for the most accurate scoring; the results are shown in Figure 5. Again, the ability to discriminate mismatches is strongly dependent on the size of both the incoming and annealing oligonucleotides, exhibiting a narrow window for mismatch discrimination. Under the conditions tested, the incoming oligonucleotide must be at least $27 \mathrm{nt}$ in length so that a D-loop is formed such that sufficient displacement of the outgoing strand is accessible for hybridization of the annealing oligonucleotide. It can be as large as $39 \mathrm{nt}$ for visualization of precise mismatch discrimination. Under these conditions, the annealing oligonucleotide must be $>13 \mathrm{nt}$ in length to form stable double D-loops, and can be as large as $25 \mathrm{nt}$; the 31-mer incoming oligonucleotide and 17-mer annealing oligonucleotide are seen to be the optimal sizes for this pair of molecules.

\section{Sequence-Specific Separation of Nucleic Acid Molecules Using Double D-Loops; a Biological Assay}

We have demonstrated that nucleic acid molecules of a known sequence can be isolated and purified in mixtures of heterologous sequences using double D-loop complexes. The assay is versatile and works with various DNA topologies. Biotinylated oligonucleotides are capable of forming stable dD-loops under the conditions that we have defined for fluorophore-labeled oligonucleotides. Because the biotinylated complex can form specifically in sequences that are homologous to the oligonucleotide, we believe that this is a useful method for allele separation, li- brary screening, and cloning. As such, we predict that clones with low representation can be isolated in a simple four-step process of invasion, binding, washing, and elution. To demonstrate this utility, pWE15 plasmids containing a kanamycinresistant gene were tested to see if they could be separated from pBR322 plasmids that contain a tetracycline-resistant gene by interacting with kanamycin (homologous) oligonucleotides. Briefly, the standard dD-loop reaction was performed using biotinylated DNA incoming and unlabeled PNA annealing oligonucleotides. As target substrates, mixtures of pWE15 plasmid were diluted serially in pBR322 plasmids so that the total DNA concentration in solution remained constant, but the ratio of pWE15:pBR322 molecules ranged from 1 to $10^{-6}$. After complex formation, the complexed nanostructures labeled with biotin were separated by binding conjugated paramagnetic beads to streptavidin, washed, and eluted. The fidelity of this process was tested by transforming the isolated plasmids into bacteria and replica plating for analyses of kanamycin and tetracycline resistance. The process successfully captured only homologous pWE15 plasmids with sensitivities down to 1 homologous molecule in a million nonhomologous to the probe sequence (Table 1). A surprisingly clean product was yielded in which rarely a contaminating pBR322 plasmid was coisolated with the specific pWE15 plasmid. The process was routinely sensitive enough to isolate specific clones represented as little as 1 in $10^{5}$ clones in a gene library.

Additionally, we have adopted this method of using short biotinylated oligonucleotide probes to isolate a particular gene variant from a background of very similar sequences. Five different plasmid targets were used: two plasmids that differ by only a single nucleotide, pWE15 $\mathrm{Kan}^{\mathrm{R}}$ carrying a wild-type kanamycin gene (TAT codon); pWE15 $\mathrm{Kan}^{\mathrm{S}}$, carrying a mutant kanamycin gene (TAG codon); and three plasmid samples with a mixture of plasmids carrying a mutant kanamycin gene (TAG codon) and a conservative replacement that confers the $\mathrm{Kan}^{\mathrm{R}}$ phenotype (TAC codon)-pWE15(CNVB1), pWE15(CNVD3), and pWE15(CNVD5). Two different preparations of these plasmid samples were used as templates for the capture assay, either su- 


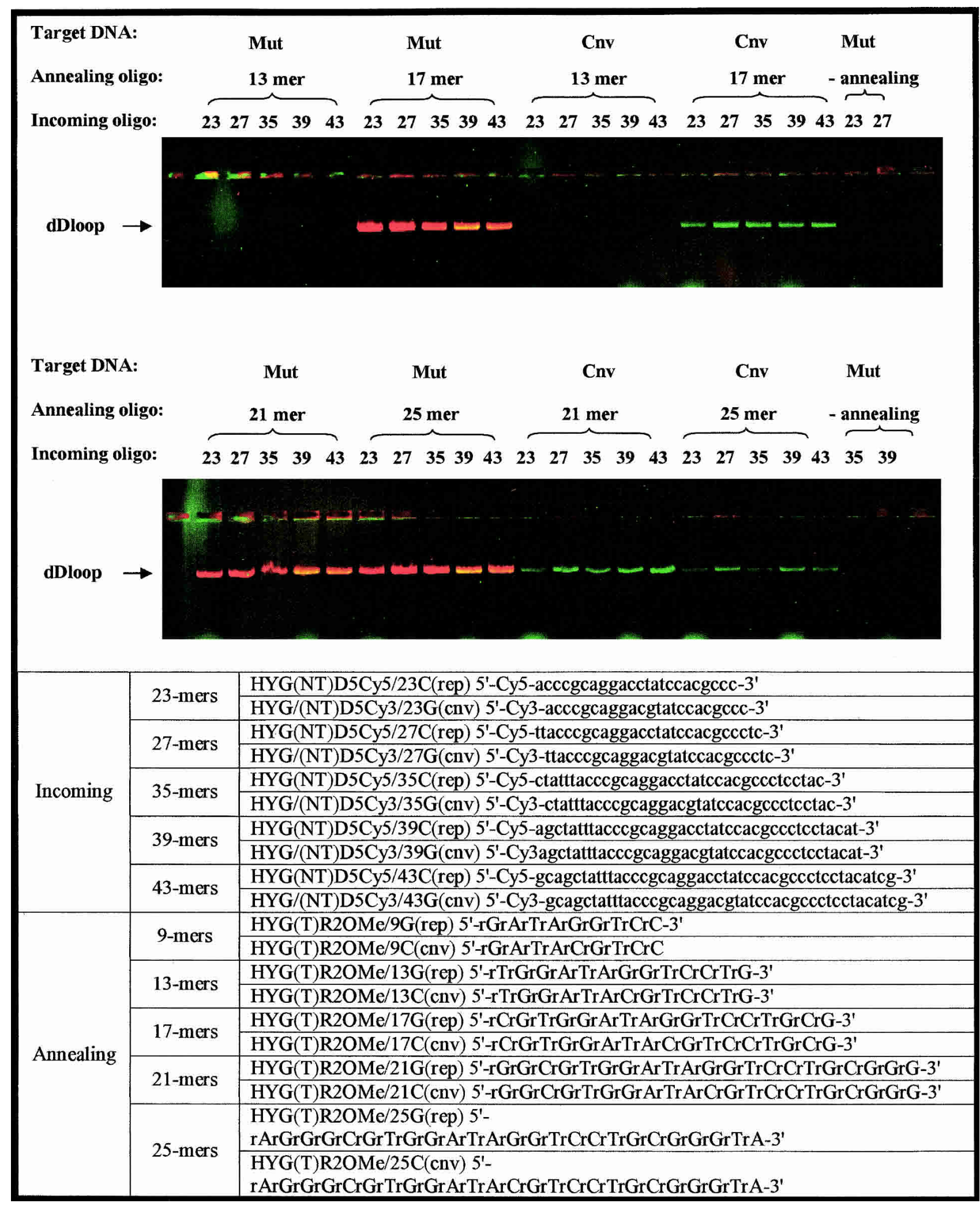

Figure 4 Size optimization of mismatch discrimination using labeled incoming oligonucleotides. The fluorescent gel picture depicts size optimization of combinations of the incoming oligonucleotides and the annealing oligonucleotides that accurately and reproducibly detect a particular gene variant. Mixed incoming oligonucleotides (exactly matching $m t$ and $c n v$ targets, which differ by a single nucleotide) and mixed annealing oligonucleotides (exactly matching $m t$ and $c n v$ targets, which differ by a single nucleotide) were used to form dD-loop complexes in either pAURHyg(wt)eGFP or pAURHyg $(w t)$ eGFP linear plasmids as indicated. Incoming oligonucleotides are labeled with Cy5 (wt) and Cy3 (cnv), and 2'-O-MeRNA annealing oligonucleotides are unlabeled. Incoming oligonucleotides 23, 252735,39 , and 43 nt in length were tested as indicated. Annealing oligonucleotides $13,17,21$, and $25 \mathrm{nt}$ in length were tested as indicated. Red bands indicate wt sequence (Cy5), green bands indicate cnv sequence (Cy3), and yellow indicates mixed signals. 


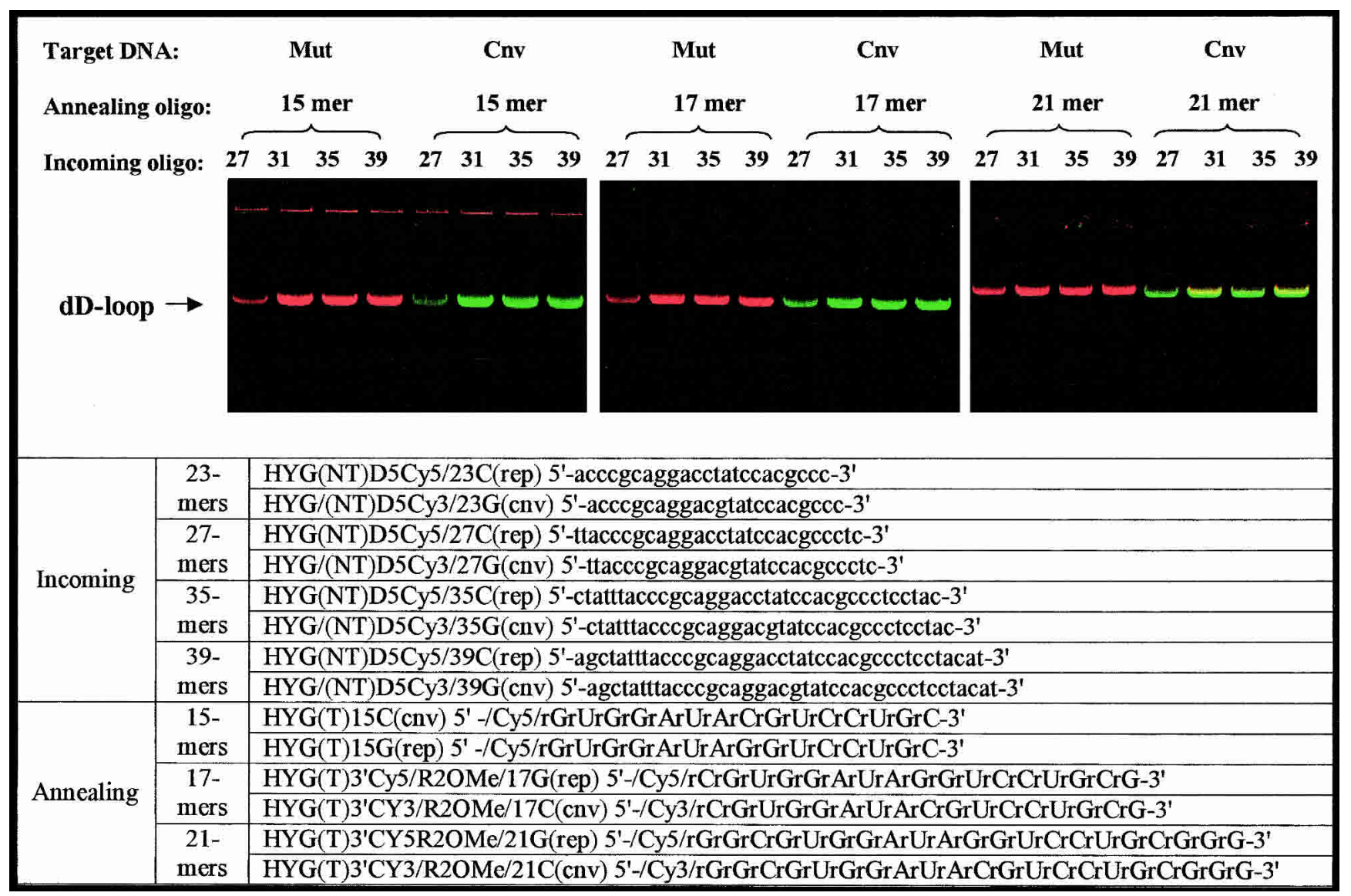

Figure 5 Size optimization of mismatch discrimination using labeled annealing oligonucleotides. The dependence of mismatch discrimination on the length of incoming and labeled annealing oligonucleotide combinations was assessed. Either linear 8-kb pAURHyg(wt)eGFP or pAURHyg(cnv)eGFP plasmids were used separately as target DNA as indicated. Mixed unlabeled incoming oligonucleotides (exactly matching $m t$ and $c n v$ targets, which differ by a single nucleotide) and mixed labeled 2'-O-MeRNA annealing oligonucleotides (exactly matching $m t$ [Cy3, red] and $c n v$ [Cy5, green] targets, which differ by a single nucleotide) were used. The ability to discriminate mismatches is strongly dependent on the size of both the incoming and annealing oligonucleotides. Incoming oligonucleotides 27, 31, 35, and $39 \mathrm{nt}$ in length were tested as indicated. Annealing oligonucleotides 15, 17, and $21 \mathrm{nt}$ in length were tested as indicated. Red bands indicate wt sequence (Cy5), green bands indicate cnv sequence (Cy3), and yellow indicates mixed signals.

percoiled or topoisomerase I relaxed cccDNA, and the ability to isolate the target converted plasmid from very similar sequences that differed by a single nucleotide was tested for fidelity. The results, shown in Table 2, reveal similarities in separation specificity as seen with our fluorescent mismatch labeling assay. We have observed that mismatched complexes can be partially stabilized in supercoiled plasmid DNA. Supercoiled plasmids that differ by a single nucleotide difference can be enriched using this method; however, there is significant coisolation of other very similar sequences. To improve fidelity, supercoiled plasmid substrates are simply relaxed by nicking or introducing positive supercoils into the target substrates. Using this additional step, coisolation is largely eliminated, yielding the capture of specific DNA variants enriched in a specific gene variant up to 20-fold.

\section{DISCUSSION}

We have developed a novel method for detecting and isolating gene variants directly from duplex DNA. The assay discriminates among single nucleotide variations in DNA substrates with various topologies without denaturation. A variety of dyesincluding Cy5, Cy3, Rox, and FAM-have been used, all of which can facilitate differential labeling for genotyping and multiplex- ing. In our studies, the fluorescent tag can be positioned on the 3 '- or 5'-end of either the incoming or annealing oligonucleotide, or any combination of these positions without loss of discrimination. We demonstrated that structures can be formed and detected by direct fluorescence on duplex DNA substrates including small PCR amplicons; linear, relaxed, or supercoiled plasmid DNAs; and preliminarily in larger substrates such as BACs, YACs, and genomic DNA (data not shown).

Linear PCR amplicons and plasmid DNA are easily genotyped by direct fluorescence using cyanine dyes, such as Cy5 and Cy3, or isolated through a biotin/streptavidin capture method. Although variants of the kanamycin and hygromycin genes were initially used to optimize the system, we have now tested several human diagnostic sequences such as TP53, k-ras, $\beta$-Globin, ApoE1, and $\beta$-tubulin in PCR amplicons with success (data not shown). We have not experienced limitations based on sequence context, but this must be examined in greater detail by a more exhaustive study. We are now developing standard protocols to achieve accurate and reproducible genotyping directly from genomic DNA of organisms with large genomes (human, murine, maize, etc.) without the need for amplification. Sensitivity is limited only by the reporter system used for detection because we have demonstrated that double D-loops form stable nanostructures in all forms of duplex DNA. 


\begin{tabular}{|c|c|c|c|c|}
\hline \multirow[b]{2}{*}{ Kan/Tet } & \multicolumn{2}{|c|}{ Colonies (presep) } & \multicolumn{2}{|c|}{ Colonies (postsep) } \\
\hline & Kan & Tet & Kan & Tet \\
\hline $1: 0$ & TNTC & 0 & $>4000$ & 0 \\
\hline 1:10 & TNTC & TNTC & 2543 & 0 \\
\hline $1: 100$ & 2060 & TNTC & 282 & 0 \\
\hline $1: 1,000$ & 1132 & TNTC & 30 & 0 \\
\hline $1: 10,000$ & 280 & TNTC & 2 & 0 \\
\hline $1: 100,000$ & 17 & TNTC & 1 & 0 \\
\hline $0: 1$ & 0 & TNTC & 0 & 0 \\
\hline 1:10 (no RecA) & TNTC & TNTC & 0 & 0 \\
\hline \multicolumn{5}{|c|}{$\begin{array}{l}\text { Shown in the table are results from a typical sequence capture ex- } \\
\text { periment. Kanamycin homologous oligonucleotides were tested to } \\
\text { see if they could separate kanamycin-resistant pWE15 plasmids from } \\
\text { tetracycline-resistant pBR322 plasmids by sequence-specific interac- } \\
\text { tion. The first column shows the ratio of pWE15 plasmids to pBR322 } \\
\text { plasmids in the experimental target mix. In the following columns, we } \\
\text { present results before separation (Colonies presep) and after the dD- } \\
\text { Loop complexes labeled with biotin were separated by binding to } \\
\text { strepavidin-conjugated paramagnetic beads (Colonies postsep). The } \\
\text { numbers presented are actual colony numbers obtained after trans- } \\
\text { forming equal samples of each condition into bacteria and replica } \\
\text { plating for kanamycin (Kan) and tetracycline (Tet) LB plates. The pro- } \\
\text { cess was routinely sensitive enough to isolate specific clones repre- } \\
\text { sented as little as } 1 \text { in } 10^{5} \text { clones in a gene library. }\end{array}$} \\
\hline
\end{tabular}

Additionally, we have demonstrated an effective method for isolating DNA fragments or clones. This process of clone isolation has enough fidelity to discriminate and isolate gene variants that differ by as little as a single nucleotide. This differs from the important method of Zeng et al. (2002) in which RecA-coated cssDNA probes are able to capture partially homologous cDNA clones representing gene variants such as paralogs and interspecies orthologs. The process can tolerate up to $35 \%$ sequence divergence from the designed probes, which conforms to the present-day doctrine of RecA enzymology in that RecA-coated single-strand DNA filaments can catalyze the strand invasion of homologous and homologous duplex DNA sequences to form hybrid intermediates. In essence, we have exploited the observation that the stability of homologous complexes is facilitated by long probes and is limited as the length is decreased. When using very short oligonucleotide probes, mismatched hybrid intermediates, although initially formed by RecA, are rapidly destabilized after being deproteinated. Routinely, precise mismatch discrimination can be achieved using incoming RecA-coated oligonucleotide filaments of $31 \mathrm{nt}$ in length and 2'-O-methyl RNAmodified annealing oligonucleotide of $17 \mathrm{nt}$ in length.

Given that several robust SNP detection assays have been developed and are already being used routinely, new genotyping technologies must possess marked advances to enter the scientific mainstream. Key attributes for such new tools include (1) a reduction in processing time; (2) cost savings in terms of reagents, equipment, and technician labor time; and (3) accuracy and reliability. The double D-loop SNP discrimination assay we describe herein meets some of these criteria. First, samples do not need to be PCR-amplified, nor do they need to be denatured prior to detection because duplex invasion is an enzymatic process. Second, reliability increases because less sample handling will be necessary to achieve results, as it will reduce false positives and negatives introduced by user error. Third, because the target sequence does not need to be denatured prior to detection, this technique is particularly useful for the in situ detection of tissue samples. Oligonucleotide-mediated detection could provide a rapid, cost-effective in situ method for more precise staging of tumors and choosing the appropriate chemotherapeutic regimen.

\section{METHODS}

\section{Oligonucleotides and DNA Substrates}

Double D-loop intermediates have been formed in linear PCR amplicons, supercoiled or linear plasmid duplex DNA substrates. Supercoiled and linear pAURHyg(x)eGFP was used as the target substrate, where " $\mathrm{x}$ " indicates a wild-type TAT codon (wt), a mutant TAG codon $(m t)$, or a converted TAC codon $(c n v)$ in the ORF of the hygromycin-resistance gene (see Liu et al. 2001). Supercoiled pAURHyg(x)eGFP plasmid DNA was purified by a QIAGEN Maxi Prep kit according to the manufacturer's protocol, and the plasmids were linearized using restriction enzyme EcoRV. These plasmids are each $7982 \mathrm{bp}$ in length, and differ only by a single nucleotide.

"Incoming" DNA oligonucleotides are designed to form RecA nucleofilaments capable of catalyzing strand invasion with duplex DNA substrates. Incoming oligonucleotides were synthesized by Integrated DNA Technologies, and contain either Cy5 or Cy3 fluorescent modifications on the 5 ' - or 3 '-ends, as indicated, and were purified by reverse phase HPLC. The "annealing" oligonucleotides are added to the reaction subsequently and designed to hybridize thermodynamically to the displaced strand created by the invading incoming oligonucleotide. Annealing oligonucleotides are designed to avoid interaction with RecA and therefore are heavily modified, composed of various modified backbones, including peptide nucleic acids (PNAs) with an acetyl group (Ac) to block a free reactive primary amine (ABI), locked nucleic acids (LNAs; Proligo), or 2'-O-MeRNA (IDT). Annealing oligonucleotides also have a Cy5 or Cy3 fluorescent modification on either the $5^{\prime}$ - or 3 '-end, as indicated in the figure Legends.

\section{In Vitro Formation of Double D-Loops: Standard Assay}

The reaction was initialized with $1.1 \mu \mathrm{L}$ of an $18 \mu \mathrm{M}$ stock of fluorescently labeled incoming oligonucleotide, $2 \mu \mathrm{L}$ of $10 \times$ Synaptic Buffer [250 mM Tris-acetate, $50 \mathrm{mM} \mathrm{Mg(acetate)})_{2}, 10$ mM DTT, $10 \mathrm{mM}$ ATP- $\gamma-\mathrm{S}$ ], water, and $12.9 \mu \mathrm{M}$ RecA, which were mixed together in a reaction volume of $18 \mu \mathrm{L}$ to preform a RecA filament on the incoming oligonucleotide. This mixture was incubated for $10 \mathrm{~min}$ at $37^{\circ} \mathrm{C}$ to allow for binding of RecA protein to the oligonucleotide (see Fig. 1). Next, the RecA-coated filament was incubated with $1 \mu \mathrm{M}$ duplex DNA in a reaction volume of $19 \mu \mathrm{L}$, and the reaction continued for $10 \mathrm{~min}$ at $37^{\circ} \mathrm{C}$; in this phase, synapsis occurs between the incoming oligonucleotide and the target nucleic acid molecule. Subsequently, $1.0 \mu \mathrm{L}$ of $36.5 \mu \mathrm{M}$ annealing oligonucleotide was added, and the reaction mixture was incubated for $2 \mathrm{~min}$ at $37^{\circ} \mathrm{C}$ to allow the annealing oligonucleotide to hybridize to the displaced strand of the duplex. The reaction was terminated by denaturing the RecA protein bound to the oligonucleotide/target complex by placing the reaction at $4^{\circ} \mathrm{C}$ on ice and adding $2 \mu \mathrm{L}$ of $10 \%$ SDS. The samples were analyzed by electroporesis through a $1.0 \%$ agarose, $1 \times \mathrm{TBE}$ gel at $4^{\circ} \mathrm{C}$, and stained with $1 \times$ SYBR Green I (FMC), a dye that binds double-stranded DNA, followed by scanning on a Typhoon imager (Amersham). The position of the double D-loop was detected by scanning for Cy5 fluorescence using an excitation wavelength of $633 \mathrm{~nm}$ and reading emission at $670 \mathrm{~nm}$, and the double-stranded target DNA was monitored by detecting the SYBR green dye using an excitation wavelength of $532 \mathrm{~nm}$ and reading emission at $526 \mathrm{~nm}$. Cy3 labels were detected using an excitation wavelength of $532 \mathrm{~nm}$ and reading emission at 555 $\mathrm{nm}$. The relative percentage of double D-loop formation can be calculated by comparing Cy3 or Cy5 intensity to SYBR Green I intensity in a complex using the ImageQuant software (Amersham).

\section{Genome Research}


Table 2. Isolation of Specific DNA Variants Using Biotinylated dD-Loops

\begin{tabular}{|c|c|c|c|c|c|c|c|c|}
\hline \multicolumn{2}{|l|}{ Target DNA } & \multicolumn{6}{|c|}{ Supercoiled plasmid } & \multirow{3}{*}{$\begin{array}{c}\text { Relaxed } \\
\text { \%Kan CNV } \\
\text { Kan/Amp }\end{array}$} \\
\hline \multirow{2}{*}{$\begin{array}{l}\text { Target } \\
\text { Plasmids }\end{array}$} & \multirow{2}{*}{$\begin{array}{c}\text { \% Kan CNV } \\
\text { (SNaP) }\end{array}$} & \multicolumn{2}{|c|}{ Colonies (presep) } & \multirow{2}{*}{$\begin{array}{c}\text { \% Kan CNV } \\
\text { Kan/Amp }\end{array}$} & \multicolumn{2}{|c|}{ Colonies (postsep) } & \multirow{2}{*}{$\begin{array}{c}\text { \% Kan CNV } \\
\text { Kan/Amp }\end{array}$} & \\
\hline & & Kan & Amp & & Kan & Amp & & \\
\hline pKan+ (tat) & - & 1124 & 1188 & $95 \%$ & 48 & 63 & $76 \%$ & $0 \%$ \\
\hline pKan - (tag) & - & 0 & 448 & $0 \%$ & 0 & 0 & $0 \%$ & $0 \%$ \\
\hline CNV B1 (tag-tac) & $5 \%$ & 60 & 570 & $10 \%$ & 53 & 128 & $42 \%$ & $>90 \%$ \\
\hline CNV D3 (tag-tac) & $69 \%$ & 363 & 437 & $83 \%$ & 164 & 183 & $90 \%$ & $>90 \%$ \\
\hline CNV D5 (tag-tac) & $10 \%$ & 90 & 520 & $17 \%$ & 28 & 38 & $74 \%$ & $>90 \%$ \\
\hline
\end{tabular}

Shown in the table are results from an adaptation of our capture method that is capable of isolating sequences that differ by as little as $1 \mathrm{nt}$. The first two columns from the left describe five different plasmid targets that were used and the starting proportion of Kan ${ }^{\text {cnv }}$ sequence in the sample: two plasmids that differ by only a single nucleotide, (1) pWE15 $\mathrm{Kan}^{\mathrm{R}}$ carrying a wild-type kanamycin gene (TAT codon): (2) pWE15 Kan ${ }^{\mathrm{S}}$ carrying a mutant kanamycin gene (TAG codon); and three plasmid samples with a mixture of plasmids carrying a mutant kanamycin gene TAG codon) and a conservative replacement (Kan ${ }^{\text {cnv }}$ ) that confers the Kan ${ }^{R}$ phenotype (TAC codon)-(3) pWE15(CNVB1), (4) pWE15(CNVD3), (5) pWE15(CNVD5). Two different preparations of these plasmid samples were used as templates for the capture assay, either supercoiled or topoisomerase I relaxed cccDNA. In each of these plasmid forms, results are presented before separation (Colonies presep) and after the biotin-labeled complexes were separated by binding to strepavidin-conjugated paramagnetic beads (Colonies postsep) for each preparation. The numbers presented are actual colony numbers obtained after transforming equal samples of each condition into bacteria and replica plating for kanamycin (Kan) and ampicillin (Amp) LB plates and the proportion of Kan to Amp (\% Kan CNV).

\section{Concurrent Detection by Differentially Labeled Incoming and Annealing Oligonucleotides}

Concurrent detection of incoming and annealing oligonucleotides was performed using a fluorescently labeled incoming oligonucleotide and a distinctly labeled annealing oligonucleotide. We used the 8-kb hygromycin linear plasmid DNA pAURHyg $(m t)$ eGFP as the target and the conditions as described for the standard reaction above. Perfectly matched 31-mer incoming oligonucleotides, one that is Cy5 labeled (5'-/Cy5/ ATTTACCCGCAGGACCTATCCACGCCCTCCT- $3^{\prime}$ ) and one that is unlabeled ( $5^{\prime}$-ATTTACCCGCAGGACCTATCCACGCCCTCCT $\left.3^{\prime}\right)$, were used as the incoming oligonucleotides. As the annealing oligonucleotides, we used 15-mer 2'-O-MeRNA oligonucleotides, one that is Cy3 labeled (5'-/Cy3/rGrUrGrGrArUrArGrGrUr CrCrUrGrC) and one that is unlabeled ( $5^{\prime}$-rGrUrGrGrArUrA rGrGrUrCrCrUrGrC).

\section{Thermodynamic Stability Study}

The standard reaction conditions were used, except that incubation temperatures of the three steps of dD-loop formation were varied. The reactions were incubated for each step of complex formation at eight temperatures: $22^{\circ} \mathrm{C}, 27^{\circ} \mathrm{C}, 32^{\circ} \mathrm{C}, 37^{\circ} \mathrm{C}, 45^{\circ} \mathrm{C}$, $50^{\circ} \mathrm{C}, 55^{\circ} \mathrm{C}$, and $60^{\circ} \mathrm{C}$. The oligonucleotides were $\mathrm{Cy} 5$ labeled and unlabeled as follows: 31-mers labeled, HYG(NT)D5Cy5/ $31 \mathrm{C}$ (rep) (5'-Cy5-atttacccgcaggacctatccacgccctcct- $\left.3^{\prime}\right)$; and unlabeled, HYG(NT)D/31C(rep)(5'-atttacccgcaggacctatccacgccctcct$\left.3^{\prime}\right)$; as the incoming oligonucleotide, and Cy5 labeled and unlabeled LNA 15-mers labeled, HYG(T)/15GrCB(5'-/Cy5/ $\left.+\mathrm{G}+\mathrm{TdG}+\mathrm{G}+\mathrm{A}+\mathrm{Td} \mathrm{A}+\mathrm{G}+\mathrm{G}+\mathrm{TdC}+\mathrm{C}+\mathrm{T}+\mathrm{G}+\mathrm{C} / \mathrm{BioTEG} /-3^{\prime}\right)$; and unlabeled, HYGLNA15T $5^{\prime}-+\mathrm{G}+\mathrm{TdG}+\mathrm{G}+\mathrm{A}+\mathrm{Td} A+\mathrm{G}+\mathrm{G}+$ $\mathrm{TdC}+\mathrm{C}+\mathrm{T}+\mathrm{G}+\mathrm{C}-3^{\prime}$ as the annealing oligonucleotide. Sets of four reactions were incubated at each of the above indicated temperatures. In the first reaction of each temperature, Cy5-labeled incoming was used alone to show the need for the annealing oligonucleotide. In the second reaction, Cy5-labeled incoming was used with an unlabeled 15-mer LNA. In the third reaction, unlabeled incoming was used with a Cy5-labeled annealing. And in the fourth reaction, the Cy5-labeled annealing was used without an incoming to show the need for the RecA filament.

\section{SNP Detection Using Labeled Incoming Oligonucleotides}

An incoming oligonucleotide mixture $(1.1 \mu \mathrm{L})$ containing 0.55 $\mu \mathrm{L}$ of $18 \mu \mathrm{M}$ Cy5-labeled Hyg(nt)D5Cy5/31C(rep) and $0.55 \mu \mathrm{L}$ of $18 \mu \mathrm{M}$ Cy3-labeled Hyg(nt)D5Cy3/31A(wt) oligonucleotides was used to detect differential stability of dD-loops with and without mismatches. The incoming oligonucleotide was coated first with RecA by incubating $2 \mu \mathrm{L}$ of $10 \times$ Synaptic Buffer [ $250 \mathrm{mM}$ Trisacetate, $50 \mathrm{mM} \mathrm{Mg(acetate)})_{2}$ and $10 \mathrm{mM}$ DTT, $\left.10 \mathrm{mM} \mathrm{ATP-} \gamma-\mathrm{S}\right]$, water, and $12.9 \mu \mathrm{M}$ RecA. This reaction was incubated for $10 \mathrm{~min}$ at $37^{\circ} \mathrm{C}$ to allow for binding of RecA protein to the oligonucleotide ("presynapsis,"; see Fig. 1 for an outline of the method). Next, RecA-coated incoming oligonucleotides were added to the duplex DNA target. The reaction was incubated for $10 \mathrm{~min}$ at $37^{\circ} \mathrm{C}$ to allow synapsis between the incoming oligonucleotide and the target nucleic acid molecule. Annealing oligonucleotide $(1.0 \mu \mathrm{L}$ of $36.5 \mu \mathrm{M})$ was then added and incubated for 2 min at $37^{\circ} \mathrm{C}$ to allow annealing of the oligonucleotide to the target nucleic acid. The reaction was stopped by denaturing the RecA bound to the oligonucleotide:target complex by placing the reaction at $4^{\circ} \mathrm{C}$ in an ice bath and adding $2 \mu \mathrm{L}$ of $10 \%$ SDS and $2 \mu \mathrm{L}$ of $10 \times$ loading dye (15\%-25\% Ficoll, optionally supplemented with $0.05 \%$ bromophenol blue). The samples were analyzed by separating by agarose gel electrophoresis, and the stability of the double D-loops under these assay conditions was monitored by detecting the fluorescent labels on the Cy3- and Cy5-labeled oligonucleotides on a Typhoon Imager as above.

\section{Size Stability Study: SNP Detection Using Labeled Incoming Oligonucleotides}

The dependence of mismatch discrimination on the length of incoming and annealing oligonucleotide combinations was also assessed. The reaction assayed $500 \mathrm{ng}$ of linear 8-kb pAURHyg (x)eGFP wild-type (wt) plasmid or "converted" (cnv) plasmid. Standard reaction conditions for forming double D-loops were used as above, with $1.1 \mu \mathrm{L}$ of $18 \mu \mathrm{M}$ mixed incoming oligonucleotides (exactly matching $m t$ and $c n v$ targets, which differ by a single nucleotide) and $1 \mu \mathrm{L}$ of $36.5 \mu \mathrm{M}$ mixed annealing oligonucleotides (exactly matching $m t$ and $c n v$ targets, which differ by a single nucleotide) used to form dD-loop complexes in either pAURHyg $(w t)$ eGFP or pAURHyg $(w t)$ eGFP linear plasmids. Incoming oligonucleotides were labeled with Cy5 ( $\mathrm{mt}$ ) and Cy3 (cnv). Annealing oligonucleotides were unlabeled and are composed of 2 '-O-MeRNA residues (indicated by " $\mathrm{r}$ " prefix). The sequences are listed in Figure 4.

\section{Size Stability Study: SNP Detection Using Labeled Annealing Oligonucleotides}

The dependence of mismatch discrimination on the length of incoming and annealing oligonucleotide combinations was assessed, with $500 \mathrm{ng}$ of linear 8-kb pAURHyg $(w t)$ eGFP plasmid or 
pAURHyg(cnv)eGFP plasmid used separately as target DNAs. Standard reaction conditions for forming double D-loops were as described above, and $1.1 \mu \mathrm{L}$ of $18 \mu \mathrm{M}$ mixed incoming oligonucleotides (exactly matching $m t$ and $c n v$ targets, which differ by a single nucleotide) and $1 \mu \mathrm{L}$ of $36.5 \mu \mathrm{M}$ mixed annealing oligonucleotides (exactly matching $m t$ and $c n v$ targets, which differ by a single nucleotide) were used. Annealing oligonucleotides were labeled with Cy3 (mt) and Cy5 (cnv) and were composed of 2'-O-MeRNA residues (indicated by " $\mathrm{r}$ " prefix). Incoming oligonucleotides were unlabeled. The sequences are listed in Figure 5.

\section{Isolation of Specific DNA Clones Using Biotinylated dD-Loops}

Double D-loop complexes were examined for sufficient stability to purify specific nucleic acid molecules of defined sequence from a complex mixture. dD-loops were formed as described above with the following exceptions. The incoming oligonucleotide, LDB/45G, has a biotin molecule attached at the 3 '-end by a TEG linker (5'-GGTGGAGAGGCTATTCGGCTAGGACTGGGCACAACAGACAATCGG-3'bioTEG), and the target nucleic acid is a mixture of supercoiled pBR322 $\left(\mathrm{Ap}^{\mathrm{R}}, \mathrm{Tet}^{\mathrm{R}}\right)$ and the supercoiled pWE15 $\operatorname{Kan}^{\mathrm{R}}$ plasmid used. Serially, limiting amounts of $250 \mathrm{ng} / \mu \mathrm{L}$ pWE15 $\mathrm{Kan}^{\mathrm{R}}$ plasmid stock were diluted in $250 \mathrm{ng} / \mu \mathrm{L}$ pBR322 $\mathrm{Tet}^{\mathrm{R}}$ plasmid stock from $100 \% \mathrm{Kan}^{\mathrm{R}}$ to a $10^{-5}$ dilution of $\mathrm{Kan}^{\mathrm{R}}$ in a Tet ${ }^{\mathrm{R}}$ background. KAN/LP/20G (AcGTGCCCAGTCGTAGCCGAAT) was the PNA annealing oligonucleotide. RecA was denatured after cooling the reaction to $4^{\circ} \mathrm{C}$ and adding $2 \mu \mathrm{L}$ of $1 \%$ SDS. $\mathrm{KCl}$ was added to a final concentration of $100 \mathrm{mM}$ to precipitate the SDS, which was pelleted at $5000 \mathrm{rpm}$ for $5 \mathrm{~min}$ in a microcentrifuge, and the supernatant was transferred to another tube. To the retained supernatant, 2 $\mu \mathrm{L}$ of prepared Dynabeads 280 diluted to a final concentration of $5 \mathrm{mg} / \mathrm{mL}$ in $2 \times \mathrm{B} \& \mathrm{~W}$ buffer (Dynal Technologies, Inc.) were added and incubated for $2 \mathrm{~h}$ at $4^{\circ} \mathrm{C}$ with rotation. The beads were then pulled from the solution by placing in a magnetic tube holder for $5 \mathrm{~min}$, and the supernatant was removed. The beads were washed three times in $1 \times \mathrm{B} \& \mathrm{~W}$ buffer by resuspending the pellets in $50 \mu \mathrm{L}$ of ice-cold buffer and agitating for $5 \mathrm{~min}$ at $4^{\circ} \mathrm{C}$, separating the magnetic Dynabeads by placing in a magnetic tube holder for $5 \mathrm{~min}$ at $4^{\circ} \mathrm{C}$, and removing the supernatant. The plasmid DNA was eluted from the Dynabeads by adding $20 \mu \mathrm{L}$ of TE and heating the solution for $15 \mathrm{~min}$ at $65^{\circ} \mathrm{C}$. Then $5 \mu \mathrm{L}$ of eluate was mixed with $20 \mu \mathrm{L}$ of electrocompetent DH10B cells (Invitrogen) and electroporated in a Cell-Porator (BRL) set at 330 $\mu \mathrm{F}, 4 \Omega$, and $400 \mathrm{~V}$. The cells were then placed in $1 \mathrm{~mL}$ of SOC medium incubated for $1 \mathrm{~h}$ at $37^{\circ} \mathrm{C}$, and $100 \mu \mathrm{L}$ of cells were spread separately on LB plates supplemented with either $10 \mu \mathrm{g} /$ $\mathrm{mL}$ tetracycline or $20 \mu \mathrm{g} / \mathrm{mL}$ kanamycin. Colonies were counted after an incubation of $\sim 16 \mathrm{~h}$ at $37^{\circ} \mathrm{C}$.

\section{Isolation of Specific DNA Variants Using Biotinylated dD-Loops}

Double D-loop complexes were examined for sufficient stability to purify specific gene variants from a complex mixture of related sequences that differ by a single nucleotide. Five different plasmid targets were used: pWE15 $\mathrm{Kan}^{\mathrm{R}}$ carrying a wild-type kanamycin gene (TAT codon), pWE15 Kan ${ }^{\mathrm{s}}$ carrying a mutant kanamycin gene (TAG codon), and three plasmid samples with a mixture of plasmids carrying a mutant kanamycin gene (TAG codon) and a conservative replacement the confers the $\mathrm{Kan}^{\mathrm{R}}$ phenotype (TAC codon)-pWE15(CNVB1), pWE15(CNVD3), and pWE15(CNVD5). Each plasmid differs by only a single nucleotide. In addition, all plasmids confer resistance to ampicillin. Prior to dD-loop separation, an aliquot of the plasmid stock for each sample was relaxed using 5 units of Topoisomerase I (Invitrogen) in $1 \times$ Synaptic Buffer and incubating for $30 \mathrm{~min}$ at $37^{\circ} \mathrm{C}$. Separation assays were performed essentially as above except us- ing KanLD5/31(TAC) (5'-GAGGCTATTCGGCTACGACTGGGCA CAACAG/3bIOteg/-3') as the incoming oligonucleotide and KAN/LP/20G (Ac-GTGCCCAGTCGTAGCCGAAT) as the PNA annealing oligonucleotide. For this, $100 \mu \mathrm{L}$ aliquots of transformed suspensions were spread separately on LB plates supplemented with either $10 \mu \mathrm{g} / \mathrm{mL}$ tetracycline or $20 \mu \mathrm{g} / \mathrm{mL}$ kanamycin. Colonies were counted after an incubation of $\sim 16 \mathrm{~h}$ at $37^{\circ} \mathrm{C}$.

\section{ACKNOWLEDGMENTS}

We acknowledge the financial support of NaPro BioTherapeutics and thank Ethan Signer for his advice.

The publication costs of this article were defrayed in part by payment of page charges. This article must therefore be hereby marked "advertisement" in accordance with 18 USC section 1734 solely to indicate this fact.

\section{REFERENCES}

Brachman, E.E. and Kmiec, E.B. 2002. The 'biased' evolution of targeted gene repair. Curr. Opin. Mol. Ther. 4: 171-176.

Burkardt, H.J. 2000. Standardization and quality control of PCR analyses. Clin. Chem. Lab Med. 38: 87-91.

Cunningham, R.P., Shibata, T., DasGupta, C., and Radding, C.M. 1979. Single strands induce recA protein to unwind duplex DNA for homologous pairing. Nature 281: 191-195.

Daly, M.J., Rioux, J.D., Schaffner, S.F., Hudson, T.J., and Lander, E.S. 2001. High-resolution haplotype structure in the human genome. Nat. Genet. 29: 229-232.

Gabriel, S.B., Schaffner, S.F., Nguyen, H., Moore, J.M., Roy, J., Blumenstiel, B., Higgins, J., DeFelice, M., Lochner, A., Faggart, M., et al. 2002. The structure of haplotype blocks in the human genome. Science 296: 2225-2229.

Gamper, H.B., Nulf, C.J., Corey, D.R., and Kmiec, E.B. 2003. The synaptic complex of RecA protein participates in hybridization and inverse strand exchange reactions. Biochemistry 42: 2643-2655.

Jayasena, V.K. and Johnston, B.H. 1993. Complement-stabilized D-loop. RecA-catalyzed stable pairing of linear DNA molecules at internal sites. J. Mol. Biol. 230: 1015-1024.

Kirk, B.W., Feinsod, M., Favis, R., Kliman, R.M., and Barany, F. 2002. Single nucleotide polymorphism seeking long term association with complex disease. Nucleic Acids Res. 30: 3295-3311.

Liu, L., Rice, M.C., and Kmiec, E.B. 2001. In vivo gene repair of point and frameshift mutations directed by chimeric RNA/DNA oligonucleotides and modified single-stranded oligonucleotides. Nucleic Acids Res. 29: 4238-4250.

Liu, L., Cheng, S., van Brabant, A.J., and Kmiec, E.B. 2002. Rad51p and Rad54p, but not Rad52p, elevate gene repair in Saccharomyces cerevisiae directed by modified single-stranded oligonucleotide vectors. Nucleic Acids Res. 31: 2742-2750.

Lizardi, P.M., Huang, X., Zhu, Z., Bray-Ward, P., Thomas, D.C., and Ward, D.C. 1998. Mutation detection and single-molecule counting using isothermal rolling-circle amplification. Nat. Genet. 19: 225-232.

Lyamichev, V., Mast, A.L., Hall, J.G., Prudent, J.R., Kaiser, M.W., Takova, T., Kwiatkowski, R.W., Sander, T.J., de Arruda, M., Arco, D.A., et al. 1999. Polymorphism identification and quantitative detection of genomic DNA by invasive cleavage of oligonucleotide probes. Nat. Biotechnol. 17: 292-296.

Millar, B.C., Xu, J., and Moore, J.E. 2002. Risk assessment models and contamination management: Implications for broad-range ribosomal DNA PCR as a diagnostic tool in medical bacteriology. J. Clin. Microbiol. 40: 1575-1580.

Morel, P., Stasiak, A., Ehrlich, S.D., and Cassuto, E. 1994. Effect of length and location of heterologous sequences on RecA-mediated strand exchange. J. Biol. Chem. 269: 19830-19835.

Parekh-Olmedo, H., Drury, M., and Kmiec, E.B. 2002. Targeted nucleotide exchange in Saccharomyces cerevisiae directed by short oligonucleotides containing locked nucleic acids. Chem. Biol. 9: 1073-1084.

Riley, J.H., Allan, C.J., Lai, E., and Roses, A. 2000. The use of single nucleotide polymorphisms in the isolation of common disease genes. Pharmacogenomics 1: 39-47.

Ryan, D., Nuccie, B., and Arvan, D. 1999. Non-PCR-dependent detection of the factor $\mathrm{V}$ Leiden mutation from genomic DNA using a homogeneous invader microtiter plate assay. Mol. Diagn. 4: 135144.

Sena, E.P. and Zarling, D.A. 1993. Targeting in linear DNA duplexes

\section{Genome Research}


with two complementary probe strands for hybrid stability. Nat. Genet. 3: 365-372.

Shi, M.M. 2002. Technologies for individual genotyping: Detection of genetic polymorphisms in drug targets and disease genes. Am. J. Pharmacogenomics 2: 197-205.

Shibata, T., Cunningham, R.P., DasGupta, C., and Radding, C.M. 1979 Homologous pairing in genetic recombination: Complexes of recA protein and DNA. Proc. Natl. Acad. Sci. 76: 5100-5104.

Smigielski, E.M., Sirotkin, K., Ward, M., and Sherry, S.T. 2000. dbSNP: A database of single nucleotide polymorphisms. Nucleic Acids Res. 28: 352-355.

Wiegand, R.C., Beattie, K.L., Holloman, W.K., and Radding, C.M. 1977. Uptake of homologous single-stranded fragments by superhelical
DNA. III. The product and its enzymic conversion to a recombinant molecule. J. Mol. Biol. 116: 805-824.

Zeng, H., Allen, E., Lehman, C.W., Sargent, R.G., Pati, S., and Zarling, D.A. 2002. Discovery of gene families and alternatively spliced variants by RecA-mediated cloning. Genomics 80: 543-551.

Zhong, X.B., Lizardi, P.M., Huang, X.H., Bray-Ward, P.L., and Ward, D.C. 2001. Visualization of oligonucleotide probes and point mutations in interphase nuclei and DNA fibers using rolling circle DNA amplification. Proc. Natl. Acad. Sci. 98: 3940-3945.

Received March 27, 2003; accepted in revised form October 9, 2003. 


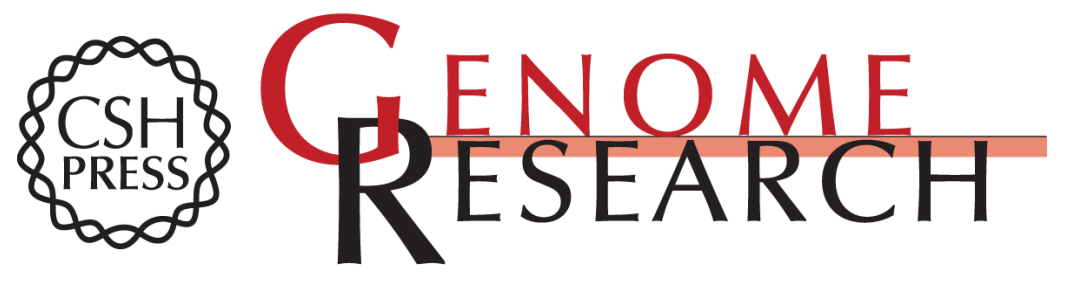

\section{Fluorescent Detection and Isolation of DNA Variants Using Stabilized RecA-Coated Oligonucleotides}

Michael C. Rice, Brandy M. Heckman, Yi Liu, et al.

Genome Res. 2004 14: 116-125

Access the most recent version at doi:10.1101/gr.1386204

References This article cites 23 articles, 5 of which can be accessed free at:

http://genome.cshlp.org/content/14/1/116.full.html\#ref-list-1

\section{License}

Email Alerting Receive free email alerts when new articles cite this article - sign up in the box at the Service top right corner of the article or click here.

\section{Affordable, Accurate Sequencing.}

